\title{
Knockdown of SLC41A1 magnesium transporter promotes mineralization and attenuates magnesium inhibition during osteogenesis of mesenchymal stromal cells
}

Yu-Tzu Tsao ${ }^{1,2}$, Ya-Yi Shih ${ }^{1}$, Yu-An Liư ${ }^{3}$, Yi-Shiuan Liü $3^{*}$ and Oscar K. Lee ${ }^{1,3,4,5^{*}}$

\begin{abstract}
Background: Magnesium is essential for numerous physiological functions. Magnesium exists mostly in bone and the amount is dynamically regulated by skeletal remodeling. Accelerating bone mass loss occurs when magnesium intake is insufficient; whereas high magnesium could lead to mineralization defects. However, the underlying magnesium regulatory mechanisms remain elusive. In the present study, we investigated the effects of high extracellular magnesium concentration on osteogenic differentiation of mesenchymal stromal/stem cells (MSCs) and the role of magnesium transporter SLC41A1 in the mineralization process.

Methods: Murine MSCs derived from the bone marrow of BALB/c mouse or commercially purchased human MSCS were treated with osteogenic induction medium containing $5.8 \mathrm{mM}$ magnesium chloride and the osteogenic differentiation efficiency was compared with that of MSCs in normal differentiation medium containing $0.8 \mathrm{mM}$ magnesium chloride by cell morphology, gene expression profile of osteogenic markers, and Alizarin Red staining. Slc41a1 gene knockdown in MSCs was performed by siRNA transfection using Lipofectamine RNAiMAX, and the differentiation efficiency of siRNA-treated MSCs was also assessed.

Results: High concentration of extracellular magnesium ion inhibited mineralization during osteogenic differentiation of MSCs. Early osteogenic marker genes including osterix, alkaline phosphatase, and type I collagen were significantly downregulated in MSCs under high concentration of magnesium, whereas late marker genes such as osteopontin, osteocalcin, and bone morphogenetic protein 2 were upregulated with statistical significance compared with those in normal differentiation medium containing $0.8 \mathrm{mM}$ magnesium. siRNA treatment targeting SLC41A1 magnesium transporter, a member of the solute carrier family with a predominant $\mathrm{Mg}^{2+}$ efflux system, accelerated the mineralization process and ameliorated the inhibition of mineralization caused by high concentration of magnesium. High concentration of magnesium significantly upregulated Dkk1 gene expression and the upregulation was attenuated after the S/c41a1 gene was knocked down. Immunofluorescent staining showed that Slc41al gene knockdown promoted the translocation of phosphorylated $\beta$-catenin into nuclei. In addition, secreted MGP protein was elevated after S/c41a1 was knocked down.

(Continued on next page)
\end{abstract}

\footnotetext{
*Correspondence: lyspub1@gmail.com; DAV47@tpech.gov.tw

${ }^{3}$ Stem Cell Research Center, National Yang-Ming University, Rm. 825,

Chih-Teh Building, No.322, Sec.2, Shih-Pai Rd, Taipei 11221, Taiwan

'Institute of Clinical Medicine, National Yang-Ming University, Taipei 11221,

Taiwan

Full list of author information is available at the end of the article
} 
(Continued from previous page)

Conclusions: High concentration of extracellular magnesium modulates gene expression of MSCs during osteogenic differentiation and inhibits the mineralization process. Additionally, we identified magnesium transporter SLC41A1 that regulates the interaction of magnesium and MSCs during osteogenic differentiation. Wnt signaling is suggested to be involved in SLC41A1-mediated regulation. Tissue-specific SLC41A1 could be a potential treatment for bone mass loss; in addition, caution should be taken regarding the role of magnesium in osteoporosis and the design of magnesium alloys for implantation.

Keywords: Mineralization, Magnesium transporter, SLC41A1, Mesenchymal stromal cells, Osteogenic differentiation

\section{Background}

Bone tissue is formed by osteoblasts, maintained by osteocytes, and broken down by osteoclasts. Physiologically, bone is constantly remodeled coordinately between bone resorption by osteoclasts and bone formation by osteoblasts [1]. Osteoblasts sustain bone mass by secreting proteins which form extracellular matrix that reinforces the strength of bone after calcium deposition [2]. Although extracellular calcification in bone cells has a positive effect for healthy bone, the ectopic calcification that occurs in soft tissues may have severe clinical consequences when localized to organs such as the arteries and kidneys [3]. However, it is still not well known how cells give rise to minerals and what the underlying regulatory mechanisms are.

Magnesium, the second most abundant intracellular free cation and the fourth most abundant metal ion in the body [4], is known for numerous physiological functions, such as enzymatic activation and Mg-ATP complex formation for energy production [5-7]. Magnesium has also been shown to inhibit Wnt/ $/$-catenin activity and reverse calcification of vascular smooth muscle cells [8]. In many of the cellular functions in which magnesium is involved, it acts as a physiological calcium antagonist; moreover, both magnesium and calcium play vital roles in bone metabolism $[9,10]$, and therefore a balance between calcium and magnesium is important in bone physiology [11]. In the human body, magnesium exists mostly in bone, either on the surface of hydroxyapatite (HAP), which is a major component of bone and teeth, or in the hydration shell around the HAP crystal [12]. Because bone serves as the reservoir for magnesium, the amount of magnesium is regulated dynamically by skeletal remodeling during bone resorption and formation for maintaining proper physiological function [13].

The very limited $\mathrm{Mg}^{2+}$ gradient across the cell membrane indicates that there is a tight regulation for $\mathrm{Mg}^{2+}$ homeostasis [14]. Several magnesium transporters have so far been identified and suggested to cooperate with each other for this tight regulation [15], yet few of them have been investigated at the molecular level. Among these transporters, solute carrier family 41 member 1 (SLC41A1) has been identified recently as a $\mathrm{Na}^{+} / \mathrm{Mg}^{2+}$ exchanger with a predominant $\mathrm{Mg}^{2+}$ efflux system containing the $\mathrm{N}$ terminus involved in $\mathrm{Mg}^{2+}$ sensing and protein kinase activities [16]. SLC41A1 has been linked with several serious human illnesses such as Parkinson's disease, preeclampsia, and nephronophthisis-like phenotypes. Moreover, SLC41A1 expression is correlated positively with the level of serum magnesium in head and neck cancer patients under chemotherapy [17]. Because serum magnesium is associated with bone mineral density and calcification, we reason that SLC41A1 may play a role in magnesium regulation for bone metabolism [11, 18]. Additionally, Slc41a1 is upregulated in some organs when mice are on a low-magnesiumcontaining diet $[19,20]$. Together with the association between Slc41a1 expression and the level of serum magnesium in mice during exercise [21], we hypothesize that SLC41A1 plays a role in magnesium homeostasis during osteogenic differentiation of MSCs.

Osteoporosis is a progressive disease resulting from an imbalance between bone deposition and resorption. Accelerating bone mass loss occurs in postmenopause animals when their magnesium intake is insufficient, and increased magnesium intake alleviates the osteoporotic symptoms [22]. On the other hand, high magnesium concentration leads to mineralization defects possibly due to magnesium substitution for calcium in the HAP structure [23]. It is reported that slow release of magnesium from scaffolds could contribute to bone regeneration in vivo $[24,25]$; whereas a hyperphysiological level of magnesium concentration inhibits extracellular matrix formation and supports chondrocyte proliferation [26]. Therefore, it is essential to understand the regulatory mechanisms in which magnesium is involved in the promotion of mineralization as well as osteoblast generation. Mesenchymal stromal cells (MSCs) possess promising potential in clinical application due to their immunomodulatory effects and the ability to give rise to various mature progenies, such as osteoblasts, adipocytes, and chondrocytes $[27,28]$. In the present study, we investigate the effect of high extracellular magnesium concentration on osteogenic differentiation of MSCs and the role of magnesium transporter SLC41A1 in the mineralization process during osteogenic differentiation. 


\section{Methods}

\section{Cell maintenance and expansion}

Mouse bone marrow-derived MSCs (mMSCs) were obtained from the femoral and tibial bone marrow of a 7-8-week-old male Balb/c mouse purchased from National Laboratory Animal Center (Taipei, Taiwan) as described previously [29]. The protocols were approved by the Taipei Veterans General Hospital Institutional Animal Care and Use Committee (IACUC 2013-048). All studies involving animals were in accordance with appropriate guidelines. The isolated cells were characterized by the surface markers using flow cytometry and assessed by the osteogenic, adipogenic, as well as chondrogenic differentiation assays before being further used for the study. Human MSCs (hMSCs) were purchased from Cell Applications, Inc. (catalog/lot number 49205a/2694; San Diego, CA, USA). This specific population of MSCs was tested for multilineage differentiation potential before being further used for the study. Cells from the 12th-15th passages used for the present study have been confirmed for their stemness prior to the experiments by checking the expression of surface markers using flow cytometry, and for their hepatogenic, osteogenic, as well as adipogenic differentiation potential. Protocols used for maintenance and expansion of mMSCs and hMSCs were described previously [30].

\section{Osteogenic differentiation of MSCs}

The basal medium used for osteogenic induction was high-glucose Dulbecco's modified Eagle's medium (HGDMEM) for mMSCs and Iscove's modified Dulbecco's medium (IMDM) for hMSCs supplemented with the induction factors: $0.1 \mu \mathrm{M}$ dexamethasone (Sigma-Aldrich), $10 \mathrm{mM} \beta$-glycerol phosphate ( $\beta$ GP; Sigma-Aldrich), and $0.2 \mathrm{mM}$ ascorbic acid (ASA; Sigma-Aldrich) [31]. The cells were seeded at 4000 cells $/ \mathrm{cm}^{2}$ and were maintained in culture medium (low-glucose DMEM supplemented with $10 \%$ FBS) for 24 hours before the induction. The induction medium was changed twice a week.

DMEM and IMDM contain $0.8 \mathrm{mM}$ magnesium ion, and therefore $0.8 \mathrm{mM}$ magnesium concentration is defined as normal magnesium concentration. The serum magnesium level in patients with renal disease is around $1 \mathrm{mM}$ [32] and a serum magnesium level around $5 \mathrm{mM}$ is associated with profound muscle weakness; on the other hand, physiological magnesium concentrations in soft tissue and bone are 8.5 and $43.2 \mathrm{mmol} / \mathrm{kg}$ wet weight respectively [15]. Therefore, for the induction medium with high concentration of magnesium, DMEM and IMDM were supplemented with $5 \mathrm{mM} \mathrm{MgCl}_{2}$ (Sigma-Aldrich) and we defined $5.8 \mathrm{mM} \mathrm{MgCl}_{2}$ as the experimental group of high magnesium concentration. A $1 \mathrm{M} \mathrm{MgCl}_{2}$ stock solution was prepared in double-distilled water $\left(\mathrm{ddH}_{2} \mathrm{O}\right)$ to make the final concentration of magnesium $5.8 \mathrm{mM}$.

\section{Quantitative real-time polymerase chain reaction} Total RNA was extracted by RNeasy Mini Kit (QIAGEN, Hilden, Germany) and reverse-transcribed to complementary DNA by MMLV High Performance Reverse Transcriptase according to the manufacturer's instructions (EPICENTRE, Madison, WI, USA). Quantitative real-time PCR (qPCR) was performed with $\operatorname{TaqMan}^{\odot}$ Fast Universal PCR Master Mix $(2 \times)$ by the Step One plus real-time PCR system (Applied Biosystems, Foster City, CA, USA) to determine the relative gene expression profiles. Primers used for qPCR are presented in Additional file 1: Table S1 with ribosomal protein S18 (Rps18) as the endogenous internal control. Relative mRNA expression was calculated by the difference of $\Delta C_{t}\left(\Delta \Delta C_{t}\right)$ of the target gene from the group without induction (day 0 ) and under osteogenic induction. The relative value RQ of gene expression was defined as $2^{-\Delta \Delta C t}$.

\section{Alizarin Red S staining and MGP detection}

For Alizarin Red staining, after washing in $\mathrm{ddH}_{2} \mathrm{O}$ three times formaldehyde-fixed cells were stained with $2 \% \mathrm{w} / \mathrm{v}$ Alizarin Red S solution ( $\mathrm{pH}$ 4.1) (Sigma-Aldrich) for 20 minutes, followed by further $\mathrm{dd}_{2} \mathrm{O}$ washing twice. Then 10\% cetylpyridinium chloride (CPC; SigmaAldrich) was used to extract the dye by incubating the stained cells at $37{ }^{\circ} \mathrm{C}$ for 20 minutes. The absorbance at $550 \mathrm{~nm}$ was measured for quantification.

For qualitative detection of mouse matrix gla protein (MGP) secreted from mMSCs in condition medium, mMSCs were cultured in 6-cm culture dishes. Fresh induction medium was added into culture dishes 3 days before being collected for the assay. MGP assay was performed by MGP ELISA kit according to the manufacturer's instruction (MyBioSource, San Diego, CA, USA) and the amount of MGP secretion was normalized by the cell number of the control group.

\section{siRNA transfection}

siRNA targeting Slc41a1 was transfected into mMSCs according to the manufacturer's instructions. Briefly, 50 nM of siRNA against Slc41a1 gene (UCGGAGUCAUCAUUGGGUCUCGAAA and UUUCGAGACCCAAUGAUGACUCCGA, Slc41a1MSS294789) was transfected with RNAiMAX transfection reagent (Invitrogen) into mMSCs for 24 hours at $37^{\circ} \mathrm{C}$. Cells were then washed with PBS and cultured in osteogenic induction medium afterward.

\section{Immunofluorescent staining}

Primary antibodies against phosphorylated $\beta$-catenin $\left(\mathrm{pSer}^{33} / \mathrm{pSer}^{37}\right.$; Abcam, UK) and phosphorylated p38 MAPK (pThr ${ }^{180} / \mathrm{pTyr}^{182}$; Cell Signaling Technology, USA) were used at 1:200 dilution according to the manufacturers' instructions. Images were taken by Olympus 
FluoView $^{\text {mi }}$ FV10i confocal microscope on the same day using the same settings of laser power and pinhole size.

\section{Statistical analysis}

All data for statistical analysis were derived from at least three independent experiments (indicated as $N$ ) and three technical repeats (indicated as $n$ ). Data were presented as mean \pm SEM. Paired Student's $t$ test was used to compare data from two groups. $p<0.05$ was considered statistically different.

\section{Results}

High concentration of extracellular magnesium inhibited mineralization of mMSCs and hMSCs

To study the effect of extracellular high concentration of magnesium on mineralization of MSCs during osteogenic differentiation, we supplied $5 \mathrm{mM} \mathrm{MgCl} 2$ in the osteogenic induction medium as the experimental group to make a total $5.8 \mathrm{mM}$ magnesium concentration and employed Alizarin Red S staining to determine the presence of calcific deposition. Calcification results from mineral deposited by the extracellular matrix which is secreted during maturation of bone cells; therefore it is an important indicator for osteogenic differentiation. Our results showed that mMSCs, which were under osteogenic induction for 12 days, had significantly reduced calcium deposition when cultured in the medium with high magnesium concentration $(5.8 \mathrm{mM})$ compared with those with physiological magnesium concentration (0.8 mM) (Fig. 1a). The morphologies of MSCs under 0.8 and $5.8 \mathrm{mM}$ magnesium concentration both turned flat during osteogenic induction (Fig. 1b). In addition, there was no significant difference in the cell numbers of MSCs differentiated under 0.8 and $5.8 \mathrm{mM}$ magnesium concentration (Fig. 1c).

We further investigated the effects of high extracellular magnesium on the expression of osteogenic marker genes of mMSCs. qPCR results showed that high concentration of magnesium significantly downregulated the gene expression of early markers including alkaline phosphatase $(A l p l)$ on day $3(p=0.01)$ and day $6(p=$ $0.016)$ after induction, osterix $(S p 7)$ on day $6(p=0.039)$,

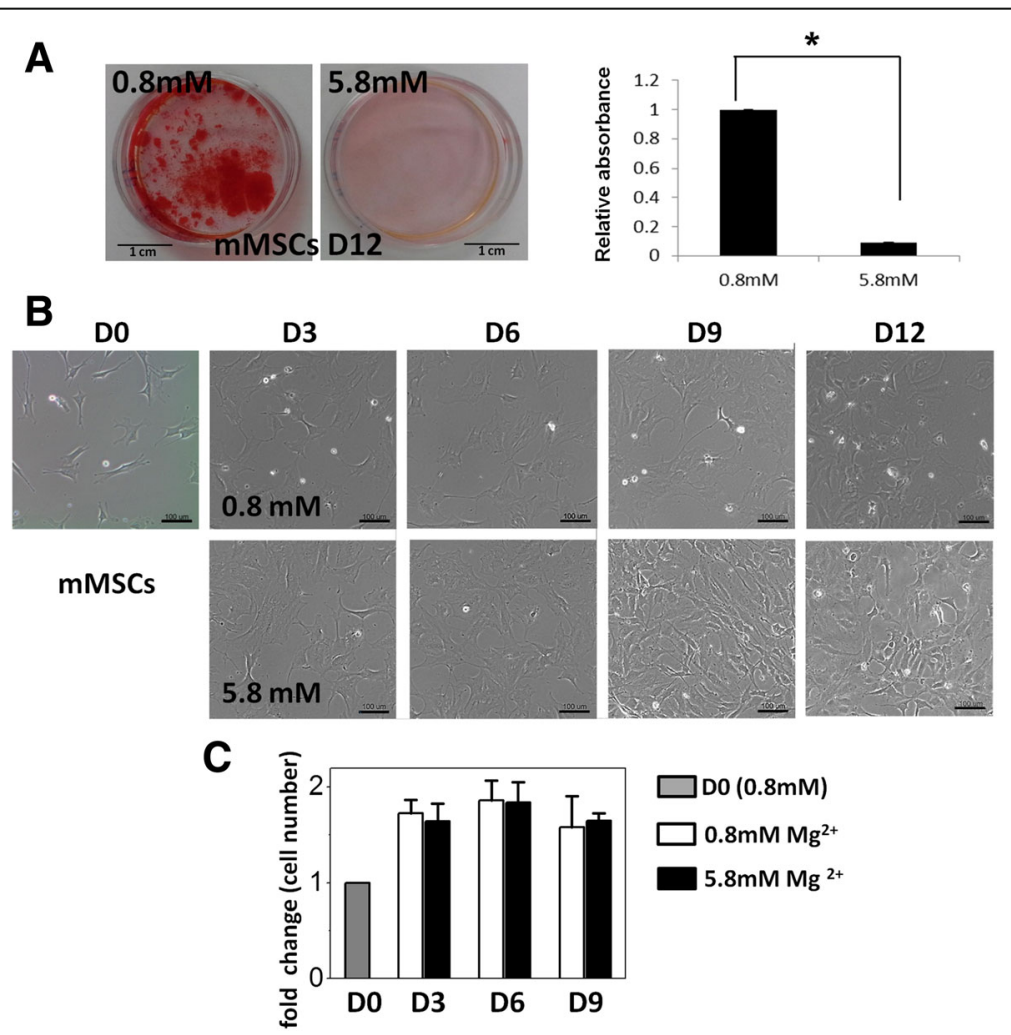

Fig. 1 High concentration of extracellular magnesium inhibited mineralization of mouse MSCs during osteogenesis. a Alizarin Red S staining images (left) and quantification (right) of mouse MSCs (mMSCs) 12 days after osteogenic induction with normal (0.8 mM) and high (5.8 mM) extracellular magnesium concentration. Biological replicate $N=3$ and technical replicate $n=3$ for every biological replicate. $\mathbf{b}$ Images of mMSCS during osteogenic differentiation with normal and high extracellular magnesium concentration. Scale bar: $100 \mu \mathrm{m}$. c Cell number of differentiating mMSCs under osteogenic induction medium containing 0.8 and $5.8 \mathrm{mM}$ magnesium for $0,3,6$, and 9 days. Cell number was determined by counting the DAPI-positive cells $(N=3, n=7)$ and normalized by cell number for day 0 . Data presented as mean \pm SEM (* $p<0.05)$. D: day 
and alpha-1 type I collagen (Col1a1) on day $6(p=0.017)$ and day $9(p=0.015)$ after the induction. On the contrary, high concentration of magnesium significantly upregulated gene expression of late markers including osteocalcin $(O c n)$ on day $3(p<0.001)$, day $6(p=0.049)$, and day $9(p=0.001)$ after induction, osteopontin $(\mathrm{Opn})$ on day $6(p=0.048)$ and day $9(p=0.048)$ after induction, and bone morphogenetic protein $2(B m p 2)$ on day $9(p=0.015)$ after induction (Fig. 2). Of note, the high fold increases of ALPL and BMP2 gene expression of differentiating MSCs compared with those of undifferentiated MSCs may be due to the extremely low expression of these two genes in undifferentiated cells (day 0).

Similarly, high magnesium concentration inhibited mineralization of hMSCs under osteogenic induction for 28 days. qPCR results showed that high concentration of magnesium significantly downregulated osterix $(S p 7)$ gene expression on day 14 and upregulated osteonectin gene expression after day 7. Magnesium had no effect on gene expression of Runx 2 and Col1A1. There is also no significant difference in cell numbers during osteogenic differentiation (more supporting data in Additional file 2: Figure S1A, S1B, S1C).
Slc41a1 knockdown promoted mineralization and attenuated magnesium inhibition

In order to identify the potential candidates that modulate the interaction of magnesium and MSCs during osteogenic differentiation, we initially screened several magnesium transporters, including SLC41A1, SLC41A2 as well as SLC41A3, and found that gene expression of SLC41A1 in differentiating MSCs was upregulated by extracellular high magnesium concentration. We hypothesized that SLC41A1 plays a role in magnesium homeostasis during osteogenic differentiation of MSCs and therefore silenced Slc41a1 gene expression to study the effect of Slc41a1 knockdown on mineralization of MSCs during osteogenic differentiation. Alizarin Red S staining demonstrated that treatment of siRNA targeting Slc41a1 promoted calcification of mMSCs. The mMSCs with Slc41a1 knockdown (KD-MSCs) exhibited calcific deposition as early as day 6 after osteogenic induction; whereas there was no calcification of the control group at the same day (Fig. 3a-c). Promotion of mineralization was also observed in human MSCs with Slc41a1 knockdown (more supporting data in Additional file 2: Figure S1E). More interesting, even when cultured in osteogenic induction medium with high concentration

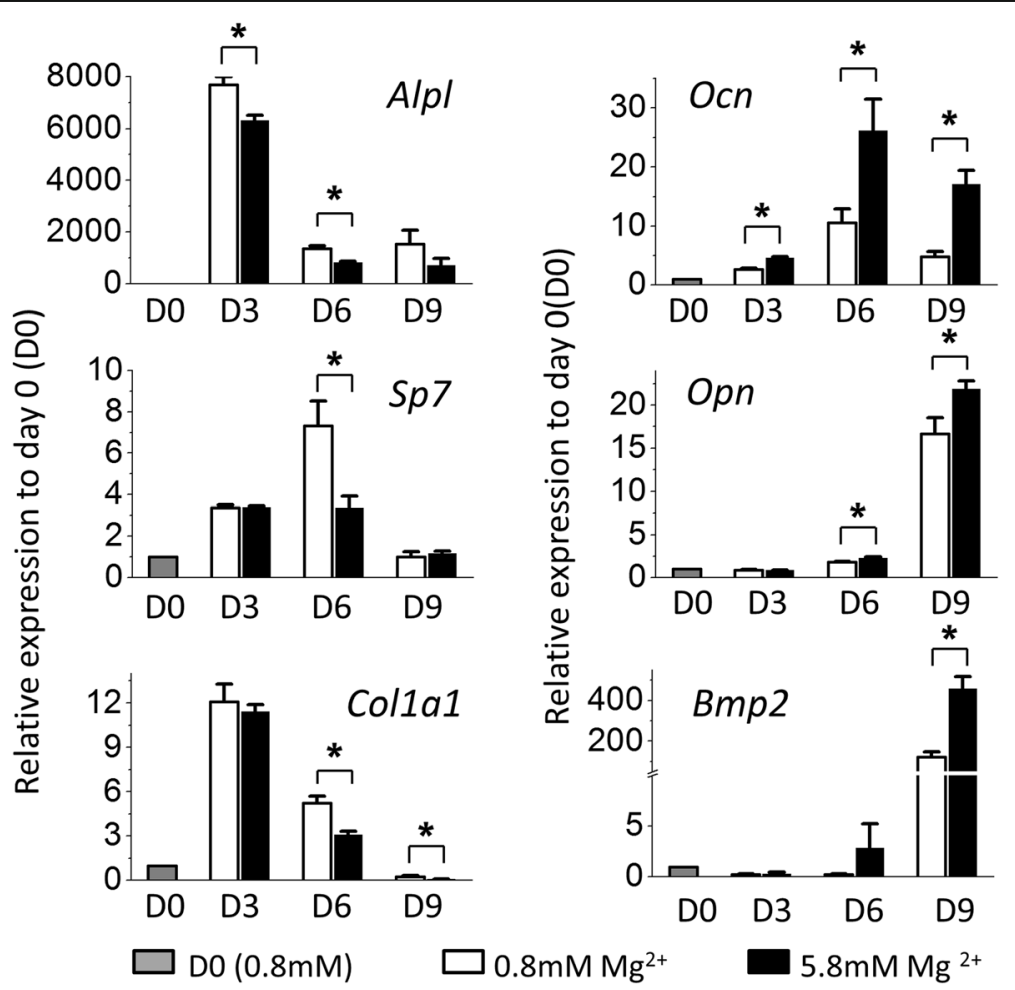

Fig. 2 High concentration of extracellular magnesium modulated osteogenic differentiation of mMSCs. qPCR results of mMSCs under osteogenic differentiation for $0,3,6$, and 9 days (D0, D3, D6, D9) with normal and high extracellular magnesium concentration. Relative gene expression indicates fold-change of gene expression in comparison with gene expression of undifferentiated MSCs on D0 $(N=3, n=3)$, presented as mean \pm SEM (* $p<0.05$ ) 
A

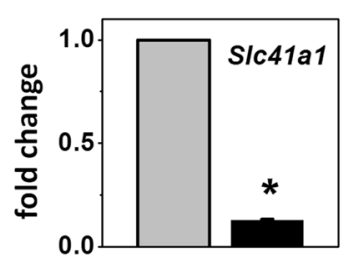

C

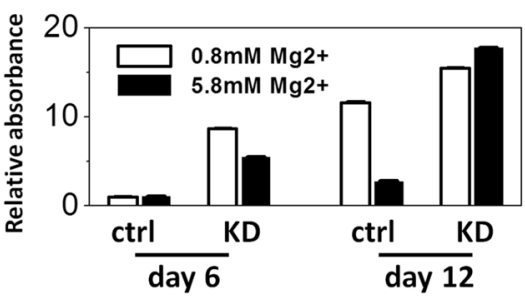

B
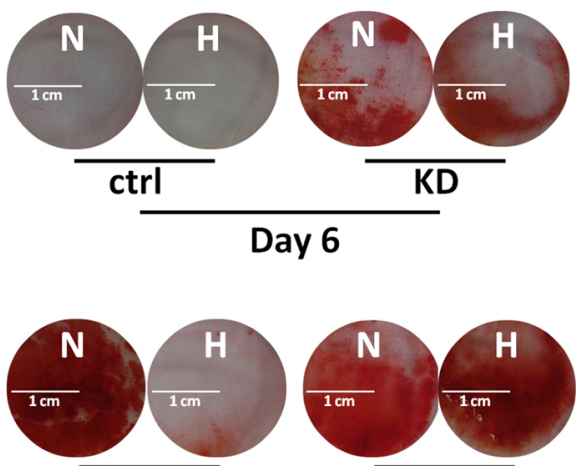

ctrl

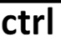

D
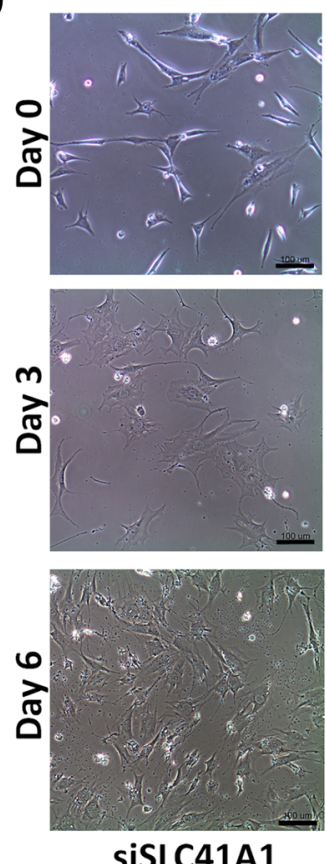

E

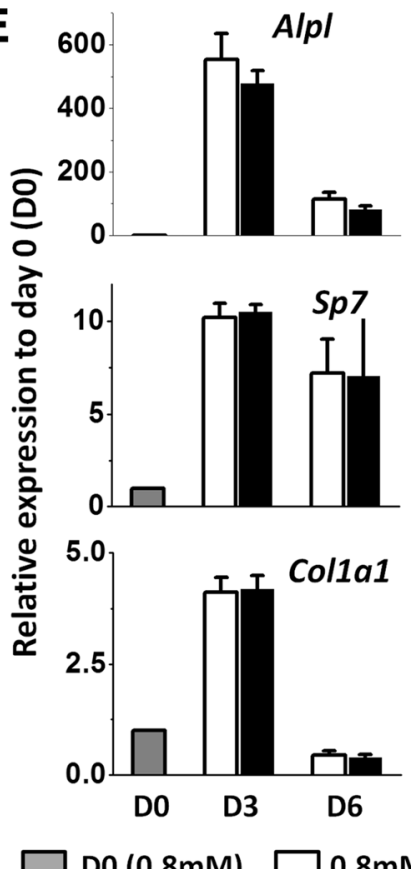

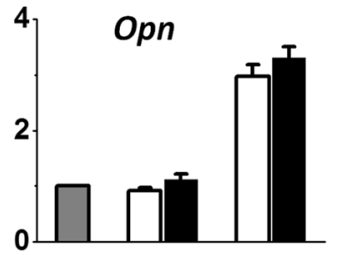
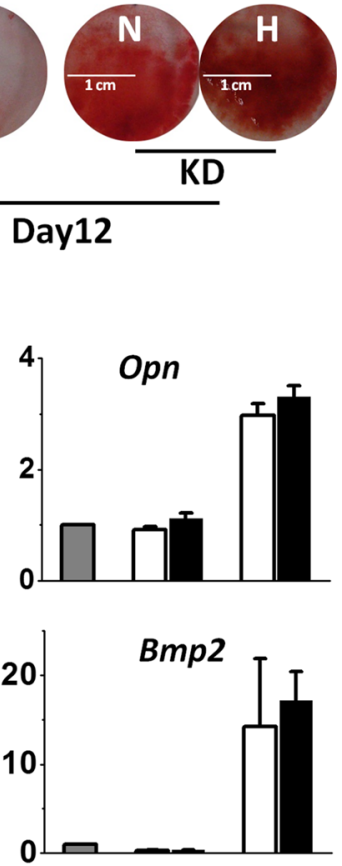

$0 . \square \mathrm{cn}$

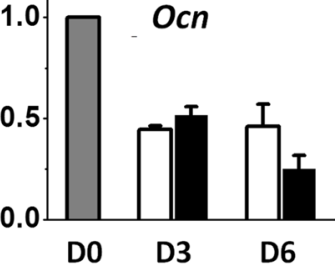

Fig. 3 S/c41a1 knockdown promoted mineralization and attenuated magnesium inhibition. a S/c41a1 expression of undifferentiated mMSCs after Slc4lal gene knockdown ( $N=3, n=3)$. b, c Alizarin Red S staining (left) and the quantification (right) of wild-type mMSCs (ctrl) and mMSCs with Slc4lal gene knockdown (KD) 6 and 12 days after osteogenic induction with normal ( $N$ : 0.8 mM) and high (H: 5.8 mM) extracellular magnesium concentration $(N=3, n=3)$. d Images of mMSCs with S/c4lal gene knockdown before and 3 and 6 days after osteogenic induction with normal magnesium concentration. Scale bar: $100 \mu \mathrm{m}$. e Gene expression of undifferentiated mMSCs with S/c41a1 gene knockdown and 3 and 6 days after osteogenic induction $(D 0, D 3, D 6)$ under 0.8 and $5.8 \mathrm{mM}$ magnesium concentration. Data normalized by the gene expression of undifferentiated Slc4la1-knockdown MSCS (DO) and presented as mean \pm SEM $(N=3, n=3)\left({ }^{*} p<0.05\right)$

of magnesium, KD-MSCs possessed similar potential for calcification as KD-MSCs cultured with physiological magnesium concentration (Fig. 3a-d). High magnesium concentration did not show significant inhibition on osteogenic differentiation of KD-MSCs. Regarding the osteogenic marker genes we tested, the levels of gene expression were similar between KD-MSCs cultured in physiological magnesium concentration and KD-MSCs in high magnesium concentration both 3 and 6 days after induction (Fig. 3e). Additionally, qPCR results revealed that even before the chemical induction (day 0), KDMSCs were likely committed into an osteogenic lineage compared with wild-type MSCs (more supporting data in Additional file 2: Figure S2). Because KD-MSCs showed an extremely high degree of mineralization after 9 days of induction, we did not further perform qPCR assays on day 9. 
Gene expression and secreted MGP were elevated after Slc41a1 was knocked down

Matrix gla protein (MGP) is known as a developmentally regulated modulator of mineralization. Our results showed that gene expression of $M g p$ increased with induction time during osteogenesis. Yet there was no significant difference between the groups cultured in normal and high magnesium concentration (Fig. 4a, b). The results demonstrated that magnesium concentration did not play a major role in regulating Mgp gene expression. We further investigated the relation of magnesium concentration and secreted MGP protein level. Even though gene expression of $M g p$ was significantly upregulated after Slc41a1 was knocked down in the early stage of the osteogenic differentiation (Fig. 4c), the results of ELISA showed that secreted free MGP protein level did not reflect the mineralization ability between wild-type and Slc41a1 knockdown groups 6 days after the induction, indicating that accumulated extracellular MGP protein was not high enough to affect mineralization on the 6th day and extracellular MGP protein was not the cause for the promotion or the inhibition of mineralization (Fig. 4d). After 9 days of osteogenic induction, only wild-type MSCs exhibited significant differences in secreted MGP level between normal and high magnesium concentration $(p=0.01)$. Because the affinity of MGP to HAP is sensitive to competitive HAP-binding protein and the surrounding ionic environment, magnesium may act as a competitor against MGP to reduce MGP-HAP binding and therefore increase the secreted unbound MGP level. Meanwhile, extracellular MGP protein secreted from Slc41a1 KD-MSCs was relatively higher than that from wild-type MSCs, indicating that MGP protein of KDMSCs was not utilized for HAP binding, possibly due to the matured mineralization of KD-MSCs, and most MGP was secreted in free form.

\section{SLC41A1 modulated Wnt/ $\beta$-catenin anti-calcifying}

\section{pathway}

Magnesium can inhibit Wnt/ $\beta$-catenin activity and reverse calcification of vascular smooth muscle cells. Because our results showed that SLC41A1 mediated the magnesium regulation of mineralization during MSC osteogenic differentiation, we therefore hypothesized that SLC41A1 is involved in the $\mathrm{Wnt} / \beta$-catenin anti-calcifying pathway. qPCR results showed that magnesium concentration did not have impact on gene expression of Wnt5a of wildtype MSCs after osteogenic induction, which is in the early stage of osteogenesis and before the onset of mineralization. However, high concentration of magnesium significantly downregulated $\beta$-catenin expression $(p=0.003)$ and the inhibition was diminished after the Slc41a1 gene was knocked down. Dickkopf-1 (DKK1) is one of the secreted antagonists of the Wnt/ $\beta$-catenin signaling pathway and its role in inhibiting mineralization has been well established. Interestingly, high concentration of magnesium significantly upregulated $D k k 1$ gene expression $(p=0.018)$ and the upregulation was attenuated after Slc41a1 gene knockdown (Fig. 5).
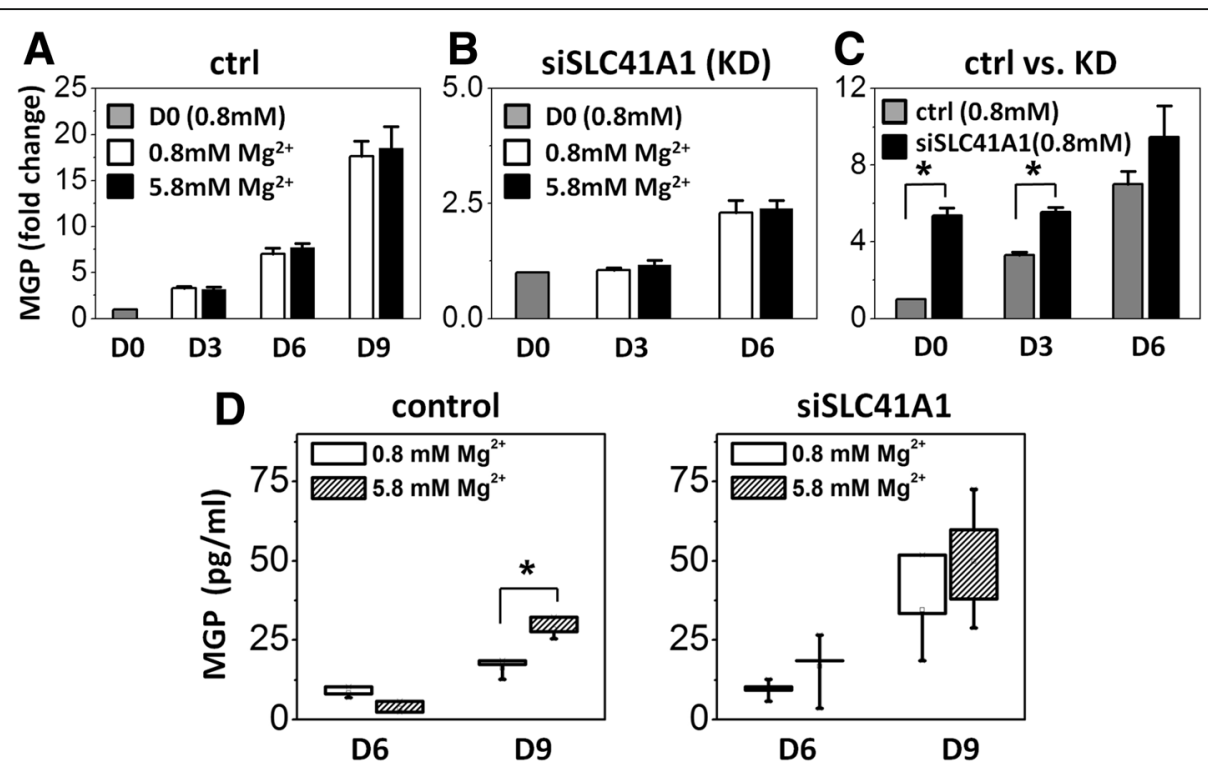

Fig. 4 Slc4la1 knockdown elevated MGP expression. a, b Mgp gene expression of control (ctrl) mMSCs and S/c41a1-knockdown mMSCs (KD) with normal and high extracellular magnesium concentration before and 3, 6, and 9 days after the osteogenic induction (D0, D3, D6, D9). Data normalized by the gene expression of undifferentiated ctrl and KD MSCs respectively (D0) $(N=3, n=3)$. c Mgp gene expression of control and Slc41a1 KD-mMSCs with normal extracellular magnesium concentration. Data normalized by Mgp gene expression of undifferentiated control MSCs (D0) $(N=3, n=3)$. d Extracellular secreted MGP concentration obtained by ELISA assay $(N=3, n=3) . M G P$ matrix gla protein. ${ }^{*} p<0.05$ 


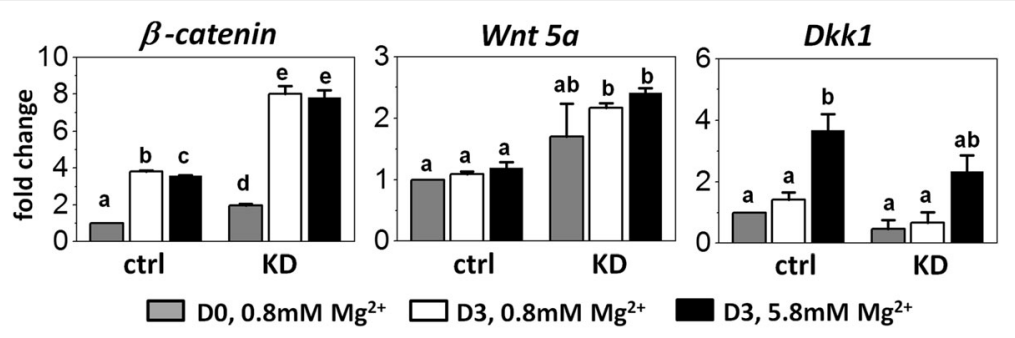

Fig. 5 S/c41al knockdown attenuated the high magnesium-induced inhibition of Wnt signaling. Gene expression of Wnt5a, $\beta$-catenin, and Dkk 1 in control (ctr) and S/c41a1 knockdown (KD) mMSCs with normal and high extracellular magnesium concentration before and 3 days after osteogenic induction ( $D 0$ and D3). Two-way analysis of variance followed by Tukey's post-hoc tests was performed for multiple comparisons. $p<0.05$ is defined statistically significant; same letters denote no significant differences. Data normalized by the gene expression of undifferentiated wildtype MSCs (D0) and presented as mean \pm SEM $(N=3, n=3)$

Results of immunofluorescent staining showed that phosphorylated $\beta$-catenin localized in the nuclei of KDMSCs 6 days after the induction; yet such nuclear localization of phosphorylated $\beta$-catenin was not observed in wild-type MSCs during this period of time. In addition, we also observed that phosphorylated p38 in KD-MSCs relocated to the cytoplasm from the nuclei on day 6 after the induction (Fig. 6). The results suggested that Wnt/ $\beta$-catenin signaling is involved in SLC41A1mediated osteogenic differentiation of MSCs.

\section{Discussion}

A recent study showed that the Slc41a1 gene expressed in mouse blood cells is downregulated immediately after exercise but returns to the basal level 24 hours after exercise [21]. The fact that the $\mathrm{Mg}^{2+}$ concentration in blood increases during short-term, high-intensity exercise [33] highlights the importance of the downregulation of this specific magnesium transport for maintaining proper physiological function. The study presented herein is the first report regarding the roles of SLC41A1 in mineralization during osteogenic differentiation. We suspect that Wnt signaling is one of SLC41A1-mediated regulation pathways for MSC osteogenic differentiation and the underlying mechanisms warrant further investigation. In addition, microarray data of MSCs in osteogenic induction medium with physiological magnesium concentration $(0.8 \mathrm{mM})$ for 3 days showed that differentially expressed genes (DEGs) between wild-type MSCs and MSCs with Slc41a1 gene knockdown were involved in focal adhesion and MAPK signaling pathways. Other DEGs of top-five hits were involved in the protein-protein interaction network of the podocyte as well as spinal cord injury (more supporting data in Additional file 2: Figure S3). The MAPK signaling pathway, focal adhesion, and Akt-mTOR signaling pathway are known to play roles in modulating osteogenic differentiation [34-37]. Whether these pathways are affected by SLC41A1-mediated magnesium ion directly or by SLC41A1-kinase activity will be investigated in the future.

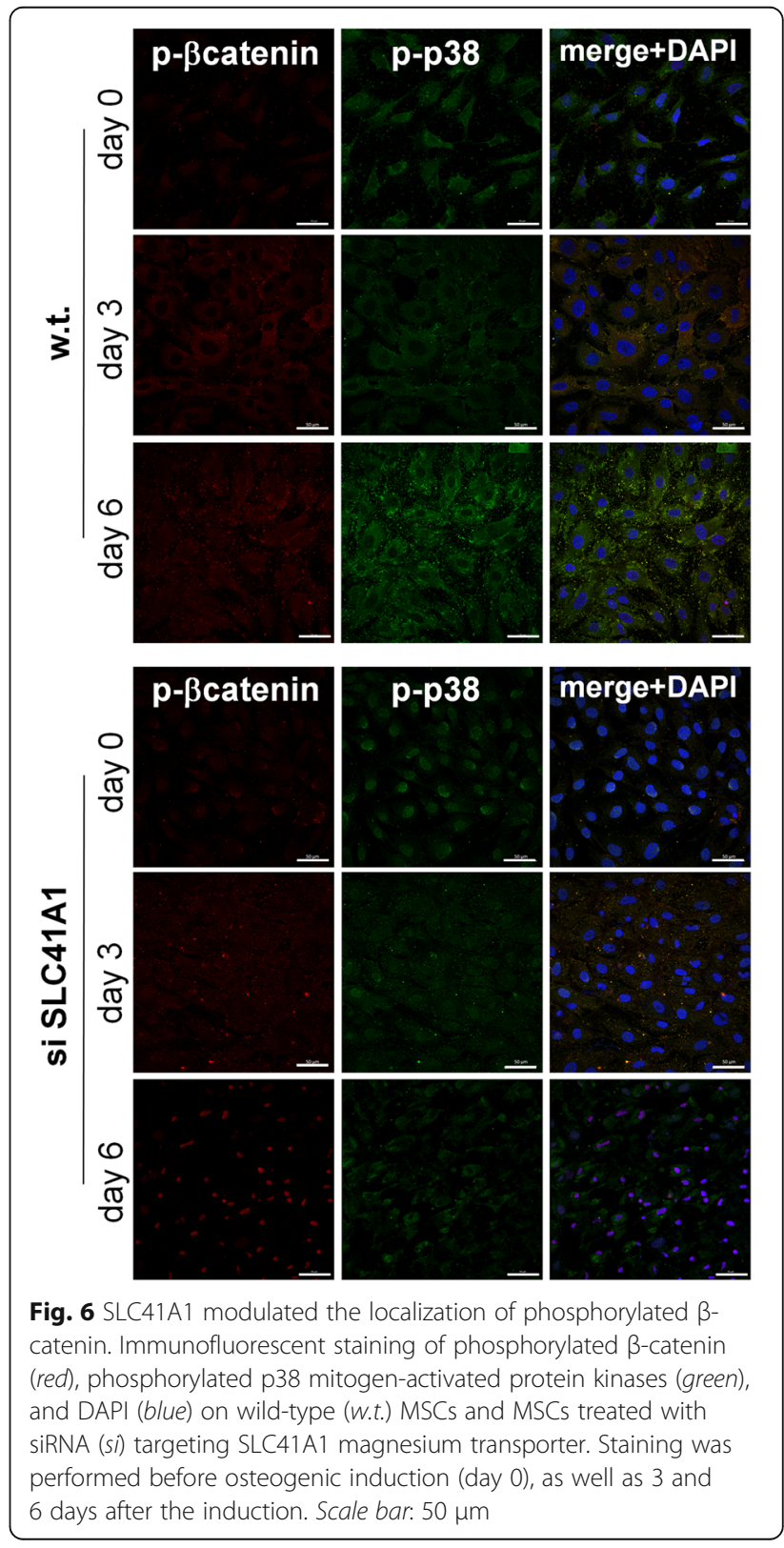


Matrix gla protein (MGP) is a developmentally regulated modulator of mineralization. Constitutive MGP expression in the chick limb has been shown to block intramembranous and endochondral ossification [38]. Mice lacking MGP die soon after birth due to the massive calcification of arterial and cartilages [39]. Although it may have other unknown effects on ectopic calcification, MGP has been suggested as a potent inhibitor of HAP crystal growth during mineralization process [40]. In addition to the competitive role of magnesium against MGP binding HAP crystal, previous study also demonstrated that high extracellular magnesium inhibits excess calcium-induced mineralization and excess calcium-induced MGP expression in prechondrogenic cell line ATDC5. Such inhibitions by excess magnesium are suggested to be mediated by inhibiting the expression of calcium-sensing receptor (CaSR) [41]. Another study also indicated that the inhibition of mineralization by high concentration of extracellular $\mathrm{Mg}^{2+}$ may be through the modulation of calcium oscillations via suppression of spontaneous ATP release and inactivation of purinergic receptors [42]. Similarly, we also observed decreased intracellular calcium concentration as well as decreased calcium influx when mMSCs were cultured in osteogenic induction medium with high concentration magnesium after 9 days (more supporting data in Additional file 2: Figure S4). This phenomenon could be explained by the competition between calcium and magnesium ions for the same transporter, such as transient receptor potential cation channel, subfamily $\mathrm{M}$, member 7 (TRPM7). TRPM7 is permeable to a number of divalent metal ions, including $\mathrm{Mg}^{2+}$ and $\mathrm{Ca}^{2+}$; it is mechanosensitive and important in osteogenesis in MSCs [43]. The results presented in our study demonstrated that SLC41A1 is important for the regulation of magnesium during mineralization; however, we cannot exclude the roles that TRPM7 and other magnesium/calcium transporters may play during MSC mineralization.

High extracellular magnesium inhibits not only mineralized matrix deposition by MSCs [42] and osteoblasts [44], but also inhibits the excess calcium-promoted mineralization in prechondrogenic cells [41]. On the other hand, it has been proposed that deficiency in magnesium causes osteoporosis. Insufficient intake of magnesium reduces the secretion of parathyroid hormone and vitamin $\mathrm{D}$, and leads to hypocalcaemia as well as the disturbance of calcium homeostasis [10]. Because parathyroid hormone also regulates bone turnover rates and the homeostasis of the related components, such as calcium and magnesium, it is not surprising that biochemical reactions involved in magnesium and bone metabolisms are complicated. Some studies showed that supplement of magnesium in daily diets could alleviate the rate of bone loss. On the other hand, our study suggests that caution should be taken when magnesium supplementation is used as a therapy for bone mass loss because high magnesium could lead to mineralization impairment.

\section{Conclusion}

High concentration of extracellular magnesium modulates gene expression of MSCs during osteogenic differentiation and inhibits the mineralization process. Additionally, we identified magnesium transporter SLC41A1 that regulates the interaction of magnesium and MSCs during osteogenic differentiation. Knockdown of SLC41A1 promotes the mineralization during osteogenesis and Wnt signaling is suggested to be involved in the SLC41A1-mediated regulation. Together, tissue-specific SLC41A1 could be a potential treatment for bone mass loss; in addition, caution should be taken regarding the role of magnesium in osteoporosis and the design of magnesium alloys for implantation because local high magnesium concentration could generate from fast degrading.

\section{Additional files}

Additional file 1: Table S1. Presenting primer sequences of mMSCs used for real-time PCR. (DOC $55 \mathrm{~kb}$ )

Additional file 2: Figure S1 showing that high concentration of extracellular magnesium inhibited mineralization of human MSCs during osteogenesis, Figure S2 showing relative expressions of osteogenic marker genes of mouse MSCs with Slc4lalgene knockdown as well as of differentiation wild-type MSCs compared with those of the undifferentiated and magnesium-untreated (0.8 mM magnesium) wild-type MSCs, Figure S3 showing the top-five pathways in which DEGs between wild-type and S/c41a1-knockdown MSCs were involved, and Figure S4 showing that high extracellular magnesium concentration decreased intracellular calcium concentration of mouse MSCs 6 days after osteogenic induction. (DOC 1139 kb)

\section{Abbreviations}

ALPL: Alkaline phosphatase; BMP2: Bone morphogenetic protein 2; COL1A1: Alpha-1 type I collagen; DKK1: Dickkopf WNT signaling pathway inhibitor 1; HAP: Hydroxyapatite; MGP: Matrix gla protein; MSC: Mesenchymal stem/stromal cell; OCN: Osteocalcin; OPN: Osteopontin; SLC41A1: Solute carrier family 41 member 1; SP7: Osterix; TRPM7: Transient receptor potential cation channel, subfamily $\mathrm{M}$, member 7

\section{Acknowledgements}

The authors acknowledge the technical services provided by the Microarray \& Gene Expression Analysis Core Facility of National Research Program for Genomic Medicine, Taiwan.

\section{Funding}

This work was supported in part by Novel Bioengineering and Technological Approaches to Solve Two Major Health Problems in Taiwan sponsored by the Taiwan Ministry of Science and Technology Academic Excellence Program under Grant Number MOST 105-2633-B-009-003. The authors acknowledge financial support from the Ministry of Science and Technology, Taiwan (MOST 103-2314-B-010-053-MY3, MOST 104-2321-B-010-008, and MOST 105-2911-I-010506). This study was also supported by Aiming for the Top University Plan, a grant from Ministry of Education.

Availability of data and materials

All data generated or analyzed during this study are included in this published article and its supplementary information files. 


\section{Authors' contributions}

Y-TT, Y-YS, Y-AL, Y-SL, and OKL contributed to the study conception, design, and data analyses. Y-SL and OKL contributed to the drafting of the manuscript. All authors approved the final version to be published and take responsibility for the integrity of the data and the accuracy of the data analysis.

\section{Competing interests}

The authors declare that they have no competing interests.

\section{Consent for publication}

Not applicable.

\section{Ethics approval and consent to participate}

The study design related to animal experiments or human tissues was approved by the ethics committee of Taipei Veterans General Hospital, Taiwan.

\section{Author details}

'Institute of Clinical Medicine, National Yang-Ming University, Taipei 11221, Taiwan. ${ }^{2}$ Division of Nephrology, Department of Medicine, Taoyuan General Hospital, Ministry of Health and Welfare, Taoyuan 33004, Taiwan. ${ }^{3}$ Stem Cell Research Center, National Yang-Ming University, Rm. 825, Chih-Teh Building No.322, Sec.2, Shih-Pai Rd, Taipei 11221, Taiwan. ${ }^{4}$ Taipei City Hospital, 145 Zhengzhou Road, Taipei 10341, Taiwan. ${ }^{5}$ Department of Medical Research, Taipei Veterans General Hospital, Taipei 11217, Taiwan.

Received: 28 September 2016 Revised: 9 January 2017 Accepted: 9 February 2017 Published online: 21 February 2017

\section{References}

1. Sims NA, Martin TJ. Coupling the activities of bone formation and resorption: a multitude of signals within the basic multicellular unit. Bonekey Rep. 2014;3:481

2. Neve A, Corrado A, Cantatore FP. Osteoblast physiology in normal and pathological conditions. Cell Tissue Res. 2011;343:289-302.

3. Persy V, D'Haese P. Vascular calcification and bone disease: the calcification paradox. Trends Mol Med. 2009;15:405-16.

4. Elin RJ. Magnesium: the fifth but forgotten electrolyte. Am J Clin Pathol. 1994;102:616-22

5. Li FY, Chaigne-Delalande B, Kanellopoulou C, et al. Second messenger role for Mg2+ revealed by human T-cell immunodeficiency. Nature. 2011;475:471-6.

6. Fawcett WJ, Haxby EJ, Male DA. Magnesium: physiology and pharmacology. Br J Anaesth. 1999:83:302-20.

7. Shechter M, Kaplinsky E, Rabinowitz B. The rationale of magnesium supplementation in acute myocardial infarction. A review of the literature. Arch Intern Med. 1992;152:2189-96.

8. Montes de Oca A, Guerrero F, Martinez-Moreno JM, et al. Magnesium inhibits Wnt/beta-catenin activity and reverses the osteogenic transformation of vascular smooth muscle cells. PLoS One. 2014;9:e89525.

9. Wu L, Luthringer BJ, Feyerabend F, et al. Effects of extracellular magnesium on the differentiation and function of human osteoclasts. Acta Biomater. 2014;10:2843-54

10. Rude RK. Magnesium deficiency: a cause of heterogeneous disease in humans. J Bone Miner Res. 1998:13:749-58.

11. Song $\mathrm{CH}$, Barrett-Connor $\mathrm{E}$, Chung $\mathrm{JH}$, et al. Associations of calcium and magnesium in serum and hair with bone mineral density in premenopausal women. Biol Trace Elem Res. 2007;118:1-9.

12. Alfrey AC, Miller NL. Bone magnesium pools in uremia. J Clin Invest. 1973; 52:3019-27.

13. Castiglioni S, Cazzaniga A, Albisetti W, et al. Magnesium and osteoporosis: current state of knowledge and future research directions. Nutrients. 2013;5: 3022-33.

14. Romani AM. Cellular magnesium homeostasis. Arch Biochem Biophys. 2011; 512:1-23.

15. Jahnen-Dechent W, Ketteler M. Magnesium basics. Clin Kidney J. 2012;5:i3-i14.

16. Schweigel-Rontgen M, Kolisek M. SLC41 transporters-molecular identification and functional role. Curr Top Membr. 2014:73:383-410.

17. Lin YJ, Cheng FC, Chien LS, et al. Expression of magnesium transporter genes in head and neck cancer patients underwent neoadjuvant cisplatinbased chemotherapy. Eur Arch Otorhinolaryngol. 2015;272:3051-7.

18. Massy ZA, Drueke TB. Magnesium and cardiovascular complications of chronic kidney disease. Nat Rev Nephrol. 2015;11:432-42.
19. Fleig A, Schweigel-Rontgen M, Kolisek M. Solute Carrier Family SLC41, what do we really know about it? Wiley Interdiscip Rev Membr Transp Signal. 2013;2(6): 10.1002/wmts.95.

20. Quamme GA. Molecular identification of ancient and modern mammalian magnesium transporters. Am J Physiol Cell Physiol. 2010;298:C407-29.

21. Tseng AP, Wei H, Hsiung $\mathrm{N}$, et al. SLC41A1, a Na/Mg exchanger, is downregulated during exercise. Biomed Rep. 2014;2:599-601.

22. Sojka JE, Weaver CM. Magnesium supplementation and osteoporosis. Nutr Rev. 1995:53:71-4

23. Roy ME, Nishimoto SK. Matrix Gla protein binding to hydroxyapatite is dependent on the ionic environment: calcium enhances binding affinity but phosphate and magnesium decrease affinity. Bone. 2002;31:296-302.

24. Staiger MP, Pietak AM, Huadmai J, et al. Magnesium and its alloys as orthopedic biomaterials: a review. Biomaterials. 2006;27:1728-34.

25. Hussain A, Bessho K, Takahashi K, et al. Magnesium calcium phosphate as a novel component enhances mechanical/physical properties of gelatin scaffold and osteogenic differentiation of bone marrow mesenchymal stem cells. Tissue Eng Part A. 2012;18:768-74.

26. Feyerabend F, Witte F, Kammal M, et al. Unphysiologically high magnesium concentrations support chondrocyte proliferation and redifferentiation. Tissue Eng. 2006;12:3545-56.

27. Jiang $Y$, Jahagirdar $B N$, Reinhardt $R L$, et al. Pluripotency of mesenchymal stem cells derived from adult marrow. Nature. 2002;418:41-9.

28. Lee OK, Kuo TK, Chen WM, et al. Isolation of multipotent mesenchymal stem cells from umbilical cord blood. Blood. 2004;103:1669-75.

29. Peister A, Mellad JA, Larson BL, et al. Adult stem cells from bone marrow (MSCs) isolated from different strains of inbred mice vary in surface epitopes, rates of proliferation, and differentiation potential. Blood. 2004;103:1662-8.

30. Teng NY, Liu YS, Wu HH, et al. Promotion of mesenchymal-to-epithelial transition by Rac1 inhibition with small molecules accelerates hepatic differentiation of mesenchymal stromal cells. Tissue Eng Part A. 2015;21:1444-54.

31. Langenbach F, Handschel J. Effects of dexamethasone, ascorbic acid and beta-glycerophosphate on the osteogenic differentiation of stem cells in vitro. Stem Cell Res Ther. 2013;4:117.

32. Meema HE, Oreopoulos DG, Rapoport A. Serum magnesium level and arterial calcification in end-stage renal disease. Kidney Int. 1987;32:388-94.

33. Bohl CH, Volpe SL. Magnesium and exercise. Crit Rev Food Sci Nutr. 2002;42: 533-63.

34. Sun C, Yuan $\mathrm{H}$, Wang $\mathrm{L}$, et al. FAK Promotes osteoblast progenitor cell proliferation and differentiation by enhancing Wnt signaling. J Bone Miner Res. 2016;31:2227-38.

35. Martin SK, Fitter S, Dutta AK, et al. Brief report: the differential roles of mTORC1 and mTORC2 in mesenchymal stem cell differentiation. Stem Cells. 2015:33:1359-65.

36. Cong Q, Jia H, Biswas $\mathrm{S}$, et al. p38alpha MAPK regulates lineage commitment and OPG synthesis of bone marrow stromal cells to prevent bone loss under physiological and pathological conditions. Stem Cell Rep. 2016;6:566-78.

37. Baker N, Sohn J, Tuan RS. Promotion of human mesenchymal stem cell osteogenesis by PI3-kinase/Akt signaling, and the influence of caveolin-1/ cholesterol homeostasis. Stem Cell Res Ther. 2015:6:238.

38. Yagami $K$, Suh JY, Enomoto-Iwamoto $M$, et al. Matrix GLA protein is a developmental regulator of chondrocyte mineralization, and when constitutively expressed, blocks endochondral and intramembranous ossification in the limb. J Cell Biol. 1999;147:1097-108.

39. Luo G, Ducy P, McKee MD, et al. Spontaneous calcification of arteries and cartilage in mice lacking matrix GLA protein. Nature. 1997;386:78-81.

40. O'Young J, Liao Y, Xiao Y, et al. Matrix Gla protein inhibits ectopic calcification by a direct interaction with hydroxyapatite crystals. J Am Chem Soc. 2011:133:18406-12.

41. Nakatani S, Mano H, Ryanghyok IM, et al. Excess magnesium inhibits excess calcium-induced matrix-mineralization and production of matrix gla protein (MGP) by ATDC5 cells. Biochem Biophys Res Commun. 2006;348:1157-62.

42. Zhang L, Yang C, Li J, et al. High extracellular magnesium inhibits mineralized matrix deposition and modulates intracellular calcium signaling in human bone marrow-derived mesenchymal stem cells. Biochem Biophys Res Commun. 2014:450:1390-5.

43. Liu YS, Liu YA, Huang CJ, et al. Mechanosensitive TRPM7 mediates shear stress and modulates osteogenic differentiation of mesenchymal stromal cells through Osterix pathway. Sci Rep. 2015;5:16522

44. Leidi $M$, Dellera F, Mariotti $M$, et al. High magnesium inhibits human osteoblast differentiation in vitro. Magnes Res. 2011;24:1-6. 